\title{
A CASE OF ARTHROGRYPOSIS MULTIPLEX CONGENITA WITH LESIONS IN THE NERVOUS SYSTEM
}

\author{
BY \\ MALCOLM FOWLER \\ From the Adelaide Children's Hospital, Adelaide, South Australia
}

(RECEIVED FOR PUBLICATION MAY 19, 1959)

Although clinical descriptions of arthrogryposis multiplex congenita are not uncommon, very few reports of the pathological findings are available. In 1932 Sheldon reviewed the literature and stated that there had been no autopsies on these cases up to that time. Since then descriptions of autopsy material have been published, including those by Price (1933), Gilmour (1946), Brandt (1947) and Adams, Denny-Brown and Pearson (1953), but the part played by abnormalities of the nervous system is not fully established by these reports. Recently, Ek (1958) has emphasized on clinical grounds the possible importance of brain damage in this disease. The present report concerns a case showing gross abnormalities in the brain and spinal cord.

\section{Clinical Findings}

The patient, a first-born female, was admitted to the Adelaide Children's Hospital at the age of 1 week. The pregnancy had been normal until the last week when, because of breech presentation, the baby had been turned. She was born as a face presentation.

On examination she was very small, weighing $4 \mathrm{lb}$. $10 \mathrm{oz}$. , but in no respiratory distress. The head circumference was $12 \frac{1}{2}$ in. and the anterior fontanelle was small. The lower jaw was asymmetrical and small. The limbs showed little spontaneous movement and all joints had a limited range of passive movement. The head was retracted and a torticollis was present, the face being turned to the left. The wrists were flexed with ulnar deviation of the hands. The fingers were extended but the metacarpo-phalangeal joints were flexed, the thumbs being adducted across the palm. The elbows were flexed. A moderate range of shoulder movements was possible, largely because of the mobility of the scapulae. The left humerus was fractured and slightly angulated near the mid-shaft; this had apparently occurred during extraction at birth. The legs showed no spontaneous movements. Although both hips and knees were kept flexed they had a moderate range of passive movement. Bilateral talipes equino-varus was present. Muscle development was very poor. A diagnosis of arthrogryposis multiplex congenita was made and because of the obscurity surrounding this clinical condition a muscle biopsy was undertaken. At operation for removal of a piece of gastrocnemius muscle great difficulty was experienced in finding anything resembling muscle tissue, and microscopical examination revealed that the material removed consisted mainly of fat and fibrous tissue with a few small bundles of tiny muscle fibres.

Under treatment in the physiotherapy department both range of movements and muscle power in the arms slowly improved but no response was obtained in the legs. After a few months she showed some ability to flex her neck when attracted by a rattle. The consultant ophthalmologist reported that there was some optic atrophy and that there was little co-ordination of eye movements. She developed little awareness of the people around her and it was concluded that she was mentally defective. Because there was no sucking ability she was fed by gavage throughout her life. During her course in hospital she had several periods of fever, accompanied by dyspnoea and signs of consolidation in the lungs, and suffered from recurrent otitis media requiring myringotomies. She died during an attack of pneumonia at the age of 11 months.

\section{Pathological Examination}

Autopsy (A.C.H. No. 120/58) was performed 36 hours after death. The body was plump and showed the deformities previously described. The anterior fontanelle was closed, the head circumference being $16 \frac{1}{2}$ in.

Muscles. The left leg was opened from the inferior iliac spine to the heel, revealing complete absence of normal muscles, which were replaced by fat. On closer inspection a few pale pinkish-yellow gelatinous strands of tissue resembling muscle bundles were found in the midthigh posteriorly and in the calf. The posterior upper part of the left arm was incised where the triceps could be easily identified, but it was very small, flabby, pale and gelatinous. . The erector spini group was slightly smaller than normal. The iliacus muscles were replaced by fat. Other muscles, such as the latissimus dorsi, the psoas, neck and abdominal muscles, were all hypoplastic, pale and soft. The diaphragm was firm but slightly pale. Microscopically, changes varied from a minimal accumulation of sudanophilic lipoid droplets in the striations to complete absence of muscles and replacement by adipose 
tissue. In one of the two sections from the buttock and thigh one small group of muscle fibres was found, most of these being very small with a few hypertrophied fibres (Fig. 1).

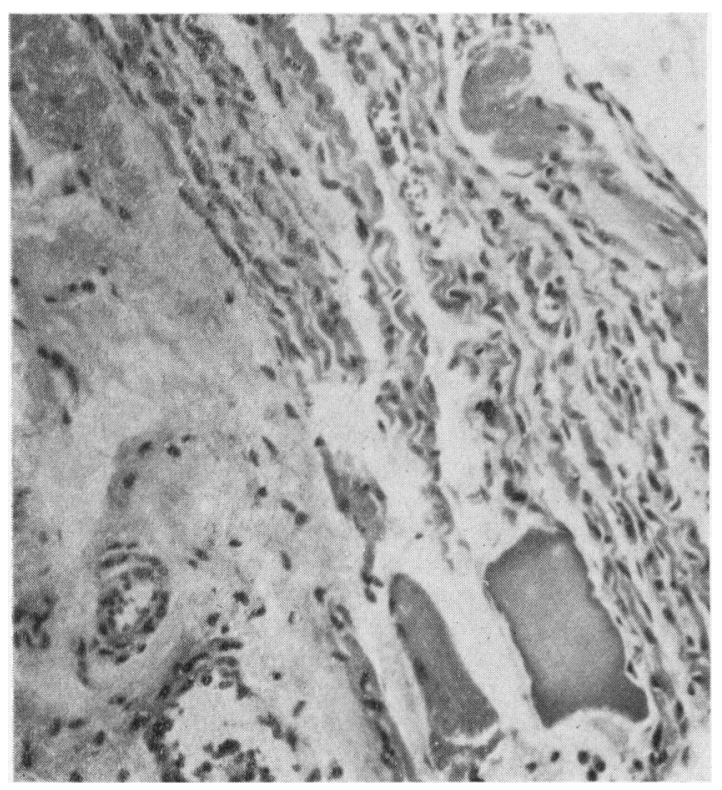

Fig. 1.-Tissue from thigh showing a group of small muscle fibres and a few hypertrophied fibres. H and E. $\times 160$.

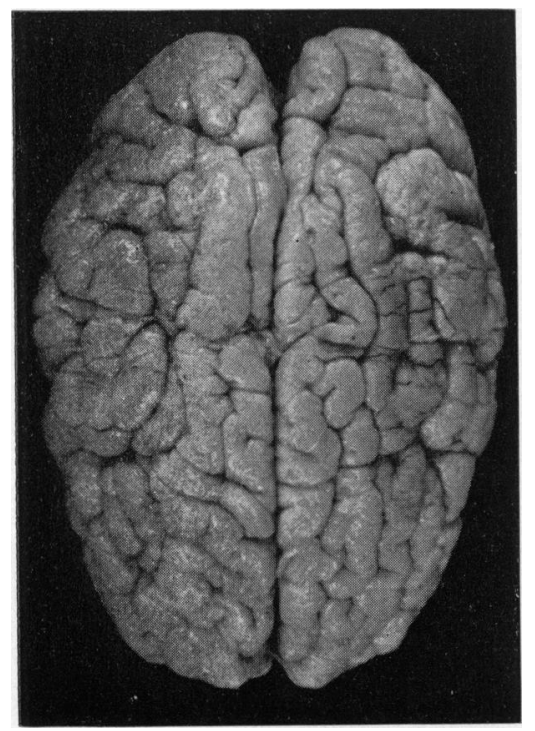

FiG. 2.-Superior surface of cerebral hemispheres showing abnormal gyral pattern.
In the less affected muscles, bundles of small fibres were very scanty but there was patchy endomysial and perimysial replacement by adipose tissue. The number of muscle spindles appeared to be increased in all muscles except in the leg specimens where none was seen, either in the small island of muscle fibres or in the surrounding adipose tissue. The diaphragm showed no abnormality apart from fatty droplets in the striations. Increase in nuclei was found only where there were groups of small fibres. There was no evidence of phagocytic activity.

The sciatic nerve appeared normal macroscopically, but microscopically there was an increase in perineural collagen and large fibres were very scanty. The only joint examined was the left knee, where the femoral articular surface was seen to be very small and flat without any curve onto the anterior and posterior surfaces of the bone. The joint capsule was normal and there were no intra-articular adhesions.

Nervous System. The brain was small, weighing $585 \mathrm{~g}$. The cerebral hemispheres were narrow and had a grossly abnormal convolutional pattern. The Sylvian fissures were short and the central sulci were not identifiable. A sulcus roughly in the region of the inferior twothirds of the central sulcus could be seen, but it did not continue to the medial edge of the hemisphere. Running along the edge of the sagittal sulcus and parallel to it were two gyri extending from the occipito-parietal region towards the frontal pole. The lateral convolution of the pair curved down and became continuous with the inferior temporal gyrus. The superior and middle temporal gyri, the parietal gyri and the posterior frontal gyri were firm, and irregular in shape, some parts being very large with smooth or finely granular surfaces, other parts being minutely convoluted or bossed. The occipital gyri, the anterior and inferior parts of the frontal lobes and the inferior temporal gyrus were relatively normal. All these cortical changes were bilateral and roughly symmetrical (Figs. 2 and 3). When the arachnoid was being stripped, it was noted that the blood vessels entered the crowns of the gyri more frequently than is normal and

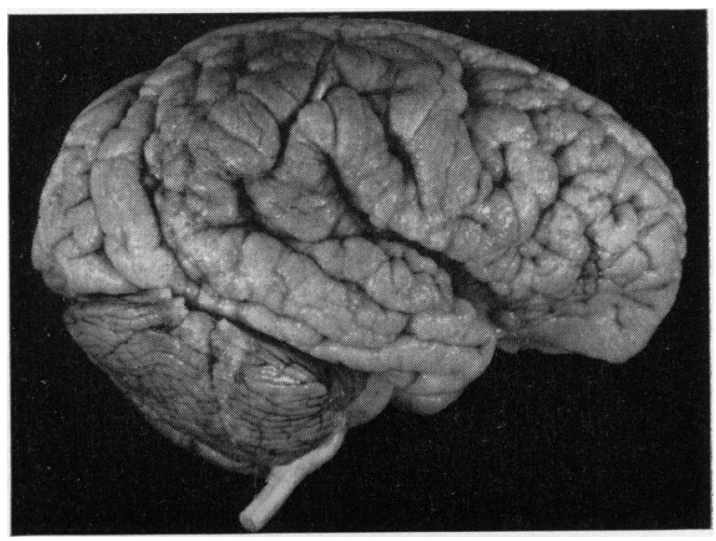

FIG. 3.-Large nodular convolutions in parietal, fronto-parietal and temporal regions. 
then ran obliquely into the cortex, particularly in the malformed gyri. The uncus was large, the optic tracts, pons and pyramidal tracts small. On incision the lateral ventricles were seen to be large and the central white matter of the hemispheres was reduced. The cortical grey matter, particularly in the areas of convolutional irregularity, was thick. Nodular masses of heterotopic grey matter projected from the lateral walls of the lateral ventricles. The thalamus was small; the hippocampus appeared normal. The spinal cord was slender and the lumbo-sacral enlargement inconspicuous.

Macroscopic examination of large coronal sections stained for myelin (Fig. 4) showed in addition a very small internal capsule containing, despite its small size, a conspicuous thalamo-cortical bundle. The internal capsule at most levels ran more horizontally than normally and the putamen was of normal size and was depressed. A few irregularly disposed islands of grey matter in the white matter of the insula apparently represented the claustrum. The temporal horn of one lateral ventricle was greatly narrowed by the protrusion of an elongated amygdaloid nucleus into it. A few small nodules of heterotopic grey matter were present under the ependyma of both temporal horns.

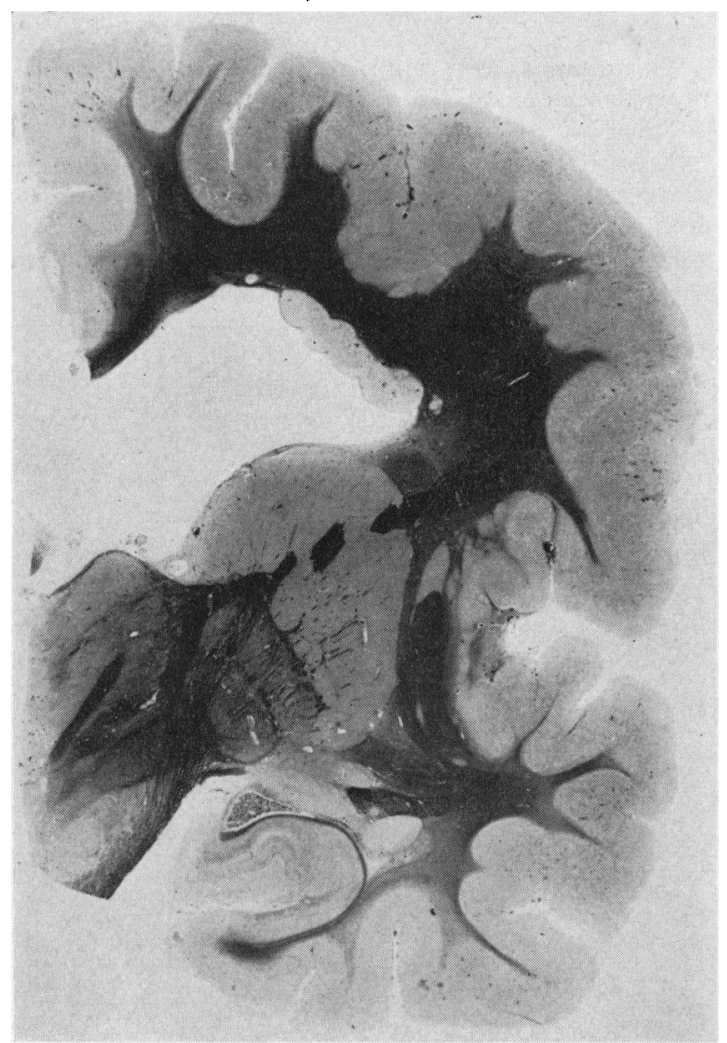

FIG. 4.-Coronal section of a hemisphere, stained by Weil's method for myelin, showing thickened areas of cortex, reduction in amount of central white matter, malformed insula and claustrum and heterotopic grey matter in wall of lateral ventricle.
Microscopically the abnormally thick cortical grey matter in the bossed areas showed no lamination although occasionally the neurones were arranged for short distances in irregular festoons. Small bundles of myelinated fibres penetrated amongst them here and there but cores of white matter did not regularly appear so that a miniature gyral pattern was not obvious. The small depressions on the pial surface were found to correspond with the site of fusion of molecular layers of adjacent abortive gyri. Sometimes the molecular layer was very thick and formed rounded masses encroaching on the underlying nerve cell layer. No giant neurones of the Betz type were found, nor were large pyramidal neurones present, the cells being small or moderate in size with relatively large nuclei. Even in areas where normal lamination of the cortical grey matter had occurred the range of variation in size of the neurones was reduced. Apart from its location, the amygdaloid nucleus was normal, as was the hippocampus.

The vessels in the abnormal cortex were numerous and often ran as quite large channels parallel to the surface. The pia contained areas of glial and fibrous thickening which often fused with the molecular layer.

There was no evidence of calcification, microglial activity or infiltration by other inflammatory cells.

Apart from the very small size of the pyramidal tracts (Fig. 5) the medulla oblongata and cerebellum were normal.

Macroscopic examination of myelin-stained sections of the spinal cord showed a reduction in size of the lateral columns, due presumably to absence of the cortico-spinal tracts. The anterior and posterior horns were therefore closer together. Lateral expansion of the anterior horns in the lumbar region was absent, the horns having an elliptical shape in cross section. The anterior columns were reduced in size but the posterior columns were normal. The substantia gelatinosa appeared to be situated just lateral to the central canal but on microscopic

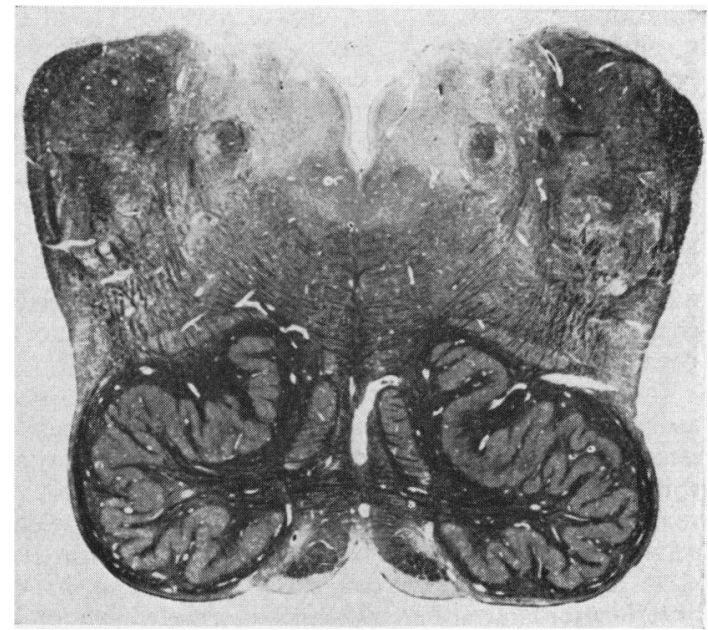

FIG. 5.-Medulla oblongata, showing small cortico-spinal tracts Weil stain $\times 5$. 


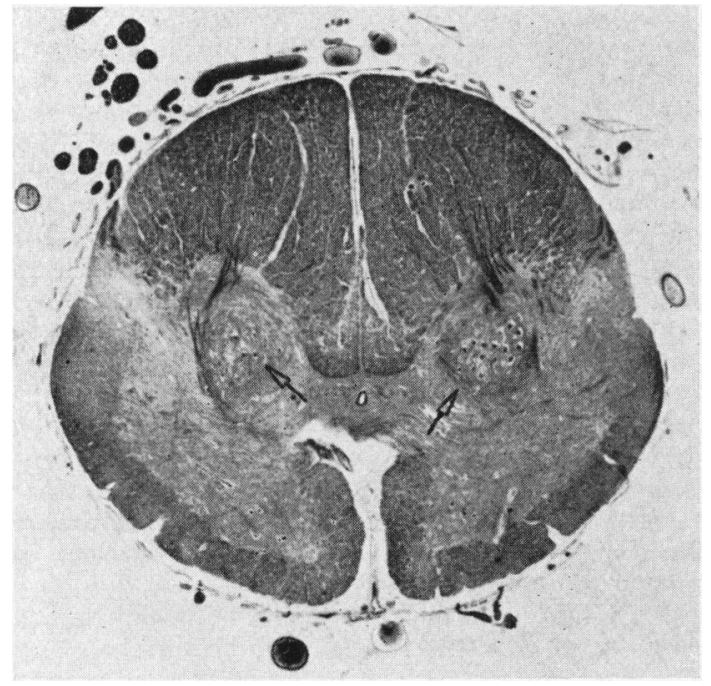

FIG. 6.-Lumbar spinal cord showing reduction in size of lateral columns, abnormal disposition of anterior and posterior horns, rounded nucleus of motor cells, indicated by arrows, and minute size of anterior nerve roots. Weil stain $\times 9$.

examination this structure was found to have been replaced by a rounded group of large motor cells (Figs. 6 and 7), the real substantia gelatinosa being compressed inconspicuously lateral to these motor neurones. In the sacral cord this group of large cells was absent, suggesting the possibility that they represented Clarke's column of the higher lumbar region, but, apart from other morphological differences, their large size seemed to exclude this. The cells of Clarke's column in the thoracic cord were normal. In the cervical region the shape of the horns was less abnormal and here a dislocated group of large motor cells was not found.

Throughout the cord the number of neurones in the anterior horns was reduced, approximating $25 \%$ of the normal population in the cervical region and showing an even greater reduction in the lumbo-sacral cord. Most of those remaining were small and angular, sometimes elongated, but with well-marked Nissl substance (Fig. 8); a few large neurones of normal size were present. There were many vacuoles in the myelin sheaths of decussating fibres in the region of the anterior horns, but apart from some central chromatolysis in the dislocated motor cells, which was consistent with post-mortem change, the bodies of the neurones showed no evidence of degeneration.

In the anterior horns there was an excess of glial tissue, mainly astrocytic, in proportion to the number of neurones present. In the position of the lateral corticospinal tracts, particularly in the thoracic and cervical regions, a few myelinated fibres were found widely separated by 'ground substance' containing scattered glial cells. No empty cell beds were found in the anterior horns and there was no abnormal sudanophilia or hyperplasia of microglia nor were perivascular cellular infiltration or capillary proliferation present.

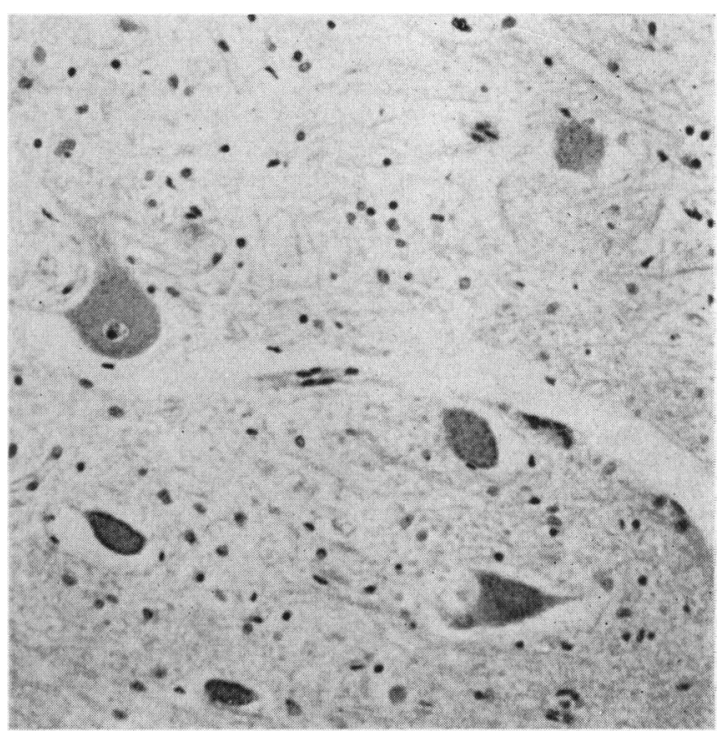

Fig. 7.-Lumbar cord showing neurones from dorsally situated nucleus. H. and E. $\times 190$.

The anterior nerve roots were very small but showed no evidence of recent demyelination.

There was a widespread bronchopneumonia, $H$. influenzae being cultured from lung swabs. Centrilobular necrosis of the liver was accompanied by an infiltration with neutrophil polymorphonuclear leucocytes and there were focal necroses in the suprarenal cortex. A blood culture was not made, but it was thought that the changes

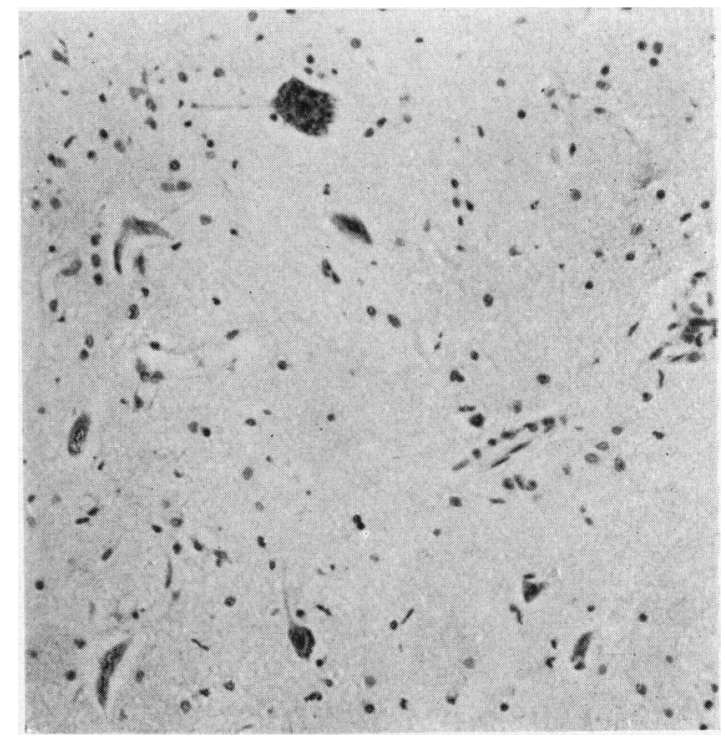

Fig. 8. - Lumbar cord showing small neurones from the most anterior part of an anterior horn. H. and E. $\times 148$ 
in the liver were most likely due to septicaemic spread of the organisms.

\section{Discussion}

Since the earlier descriptions of arthrogryposis multiplex congenita (Stern, 1923), accounts of the condition have been uniform as far as the main clinical aspects are concerned. The affected child is born with a restricted range of movement in multiple joints, usually bilateral. The degree and distribution of the deformities are variable, but a common pattern is seen in extension of the elbows, ulnar deviation of the hands and flexion of the fingers, flexion of the hips and talipes equino-varus. Dislocation of the hips is common and fractures of the limbs, mostly resulting from difficult extractions at birth, have frequently been reported. Muscular development, particularly in relation to the most severely affected joints, is poor, and muscle biopsy has shown small muscle fibres and occasionally some adipose tissue replacement. Often the mother has noticed that foetal movements were slight or absent. When information is sought on the occurrence of neurological changes and mental deficiency, on the other hand, it is only in the last two decades that clinical observations implicating the nervous system have begun to accumulate (Ek, 1958).

The interpretation of the muscular changes and of the lesions in the central nervous system has been delayed to a large extent by the lack of a complete autopsy. Price (1933) found the brain normal whereas sections of the spinal cord showed degeneration of the neurones in both anterior and posterior horns and also degenerative changes in the white columns, but her photographs do not illustrate these points with any certainty. The arm muscles were reduced in size and intermingled with fatty tissue; some were absent. Dissection of the elbow joint revealed circumscribed articular surfaces on the lower end of the humerus. Gilmour's case (1946), a foetus, stillborn at 30 weeks' gestation, was studied in more detail. He found 'slight hydrocephalus of unexplained cause', a reduction in the number of anterior horn cells in the thoracic cord and a reduction in size of those in the seventh cervical segment. Most muscles were greatly reduced in size; some were absent. The articular surfaces of the knee joint were smaller than normal.

Brandt (1947) found no gross structural alterations in the central nervous system, but there were 'profound' changes in the motor neurones of the anterior horns of the spinal cord. As pointed out by Adams et al. (1953), the changes shown in the illustrations could be due to post-mortem artefacts. Biopsy of a quadriceps muscle in Brandt's case showed extreme atrophy. The muscles were not apparently examined microscopically post mortem, and no joints were mentioned.

Adams et al. (1953) reported two cases, one being incompletely examined. In one case they found a disappearance of anterior horn cells in the lumbosacral region and a reduction in their number and size at higher levels. The brain was small and had an abnormal convolutional pattern and large lateral ventricles, but further details of the brain are not given. In their other case they found a reduction in the number and size of anterior horn cells in the lumbo-sacral segments of the spinal cord.

Kanof, Aronson and Volk (1956) described three patients, one of whom came to autopsy. The muscles were in various degrees reduced in size; one knee joint was examined and found to be normal. A normal number of anterior horn cells was seen in the lumbar cord, but a decrease in number had occurred in the lower cervical region. Their photograph of the lumbar cord, illustrating what they have interpreted as demyelination of the lateral corticospinal tracts, could represent absence or reduction of the tracts with disturbed relations of the anterior and posterior horns.

One of the cerebral abnormalities found in the present case has been called 'micropolygyria' (Norman, 1958), the abnormal cortex consisting of large gyri often with a granular surface resembling cobblestoning. In some cases the internal arrangement suggests incompletely separated small gyri, but in this case, although there was fusion of the molecular layers, the thick zone of neurones was rarely penetrated by miniature cores of white matter. In addition, as is sometimes found with micropolygyria, nodules of grey matter were present in the walls of the lateral ventricles. The large fissures of the brain were abnormal, the central sulcus, if present at all, being incomplete. No Betz cells were identifiable and the pyramidal tracts were minute. Apart from deducing that these findings are due to an arrest of neuronal migration with subsequent abnormal development, there is nothing to indicate whether the aetiology is genetic or acquired. Except for occasional foci of pial thickening by glial and, to a lesser extent, fibrous tissue there was nothing which could reasonably be construed as evidence of an inflammatory reaction, nor were there any remains of past softening or haemorrhage. Bertrand and Gruner (1955), in a detailed description of micropolygyria, which they call 'status verrucosus', emphasize the abnormality in the course of the small cortical blood vessels such as that observed in the present case, and Norman (1958) mentions that this finding is compatible with 
Bielschowsky's view (1917) that micropolygyria is a consequence of abnormal vascularization of the cortex.

The findings in the anterior horns indicated a loss of neurones, with hypoplasia of a large proportion of the remainder. Since the posterior horns merged with the anterior horns it was not certain how many of these small neurones were rightful members of the anterior horns, but their angular shape and welldeveloped Nissl substance indicated that most of them were of motor type. Although the abnormal disposition of the horns of the spinal cord may be attributed to the virtual absence of the lateral cortico-spinal tracts, the abnormality of the anterior horn cells is unlikely to be an example of transsynaptic atrophy, but rather a defect in development contemporaneous with that in the brain. That the absence of the cortico-spinal tracts does not necessarily lead to congenital muscular hypoplasia is evident from the well-developed musculature in anencephalic monsters.

Adams et al. (1953) have pointed out that arthrogryposis multiplex congenita might well have a multiple aetiology. Recently Banker, Victor and Adams (1957) reported two cases which were due to muscular dystrophy and they refer to several others reported in the literature. In spite of the small number of cases submitted to autopsy it is evident that lesions in the nervous system may also produce this clinical syndrome, that under such conditions the muscle changes are secondary to neural abnormalities, and that the changes in the joints can be attributed, as Gilmour suggested (1946), to lack of movement during the early stages of their development in utero.

\section{Summary}

A female baby was born with arthrogryposis multiplex congenita; she never sucked and there were no spontaneous movements of the legs. Mental development was thought to be impaired and there was some optic atrophy. Autopsy at the age of 11 months revealed micropolygyria, hypoplasia of the cortico-spinal tracts and a great reduction in the number of anterior horn cells. Voluntary muscles were virtually absent in the legs and small elsewhere, the microscopic appearances being consistent with neurogenic atrophy.

The pathological findings in some previously reported cases are briefly reviewed. Although the aetiology of arthrogryposis multiplex congenita is multiple, it is concluded that lesions in the nervous system account for a significant proportion of cases.

Thanks are due to the Board of the Adelaide Children's Hospital and to Dr. Eric Sims for permission to publish details of this case. I am grateful to Mrs. Helga Thiede for technical assistance and to $\mathrm{Mr}$. R. Boyd for the preparation of the illustrations.

\section{REFERENCES}

Adams, R. D., Denny-Brown, D. and Pearson, C. M. (1953). Diseases of Muscle. Hoeber, New York.

Banker, B. Q., Victor, M. and Adams, R. D. (1957). Brain, 80, 319. Bertrand, I. and Gruner, J. (1955). J. Neuropath. exp. Neurol., 14, 331 .

Bielschowsky, M. (1917). J. Psychol. Neurol (Lpz.), 22, 1. Quoted by Norman, R. M. (1958).

Brandt, S. (1947). Acta paediat. (Stockh.), 34, 365

Ek, J. I. (1958). Ibid., 47, 302.

Gilmour, J. R. (1946). J.Path. Bact., 58, 675.

Kanof, A., Aronson, S. M and Volk, B. W. (1956). Pediatrics, 17, 532.

Norman, R. M. (1958). In Neuropathology, by Greenfield, J. G. et al. Chapter 5. Arnold, London.

Price, D. S. (1933). Arch. Dis. Childh., 8, 343.

Sheldon, W. (1932), Ibid., 7, 117.

Stern W. G. (1923). J. Amer. med. Ass., 81, 1507. 